03,09

\title{
Линейно-циркулярный дихроизм в полупроводнике со сложной валентной зоной с учетом четырехфотонного поглощения света
}

\author{
(C) Р.Я. Расулов, В.Р. Расулов, И.М. Эшболтаев \\ Ферганский государственный университет, \\ Фергана, Узбекистан \\ E-mail: r_rasulov51@mail.ru
}

(Поступила в Редакцию 24 мая 2016 г.)

\begin{abstract}
Теоретически исследован линейно-циркулярный дихроизм многофотонного поглощения света в полупроводниках со сложной валентной зоной.

Рассчитаны матричные элементы оптических переходов, протекающих между подзонами валентной зоны полупроводника типа $p$-GaAs. При этом учтены переходы, связанные как с неодновременным поглощением отдельных фотонов, так и одновременным поглощением двух фотонов. Получено выражение для температурной зависимости коэффициента многофотонного поглощения поляризованного излучения, где учтены оптические переходы между подзонами тяжелых и легких дырок полупроводника.
\end{abstract}

DOI: 10.21883/FTT.2017.03.44153.215

Нелинейное поглощение света в полупроводнике с вырожденной валентной зоной, обусловленное прямыми оптическими переходами между подзонами тяжелых и легких дырок и зависящее от состояния поляризации излучения, исследовано в [1-8]. В этих работах считается, что нелинейность в зависимости коэффициента однофотонного поглощения от интенсивности возникает за счет резонансного насыщения поглощения. Это насыщение обусловлено фотоиндуцированным изменением функций распределения легких и тяжелых дырок в области импульсного пространства вблизи поверхности $E_{\mathrm{hh}}(\mathbf{k})-E_{\mathrm{lh}}(\mathbf{k})-\hbar \omega=0$, соответствующей условию резонанса. Здесь $E_{\mathrm{hh}}(\mathbf{k})\left(E_{\mathrm{lh}}(\mathbf{k})\right)-$ энергетический спектр тяжелых (легких) дырок, $\omega$ - частота света.

В работах [8-16] исследовано многофотонное поглощение света в полупроводниках. В [8] исследован многофотонный линейно-циркулярный дихроизм (ЛЦД) в $p$-Ge в режиме развитой нелинейности, когда в поглощение вносят сопоставимый вклад $n$-фотонные процессы с $n=(1-5) .{ }^{1}$ В работах [9-16] исследованы двух-, трехфотонные процессы в полупроводниках, обусловленные оптическим переходами между валентной зоной и зоной проводимости, однако в них, во-первых, не учтен вклад эффекта насыщения поглощения, а во-вторых, не учтены оптические переходы, обусловленные одновременным поглощением двух фотонов.

Здесь мы рассмотрим четырехфотонное поглощение поляризованного излучения как с учетом эффекта когерентного насыщения поглощения, так и без его учета. Поэтому далее рассматривается многофотонное поглощение поляризованного излучения в полупроводниках типа $p$-GaAs, обусловленное прямыми оптическими переходами между подзонами легких и тяжелых дырок. При этом считается, что энергия фотона $\hbar \omega$ удовле-

\footnotetext{
1 Линейно-циркулярный двухфотонный дихроизм предсказан в [1].
}

творяет неравенству $\hbar \omega \ll E_{g}, \Delta_{\text {sо }}$, где $E_{g}-$ ширина запрещенной зоны, $\Delta_{\text {so }}-$ спин-орбитальное расщепление валентной зоны. При этом оптическое поглощение можно рассматривать в рамках эффективного гамильтониана $H(\mathbf{k})$ [5-8], заменяя в нем $\mathbf{k}$ на

$$
\mathbf{k}=-i \boldsymbol{\nabla}-\frac{e}{c \hbar} \mathbf{A},
$$

где $\mathbf{A}=A_{0}\left(\mathbf{e} e^{i \mathscr{N} \mathbf{r}-i \omega t}+\mathbf{e}^{*} e^{-i \mathscr{N} \mathbf{r}+i \omega t}\right), A_{0}-$ амплитуда векторного потенциала электромагнитной волны, $\mathscr{N}$ волновой вектор фотона.

Ввиду малости волнового вектора фотона по сравнению с волновым вектором электрона (дырки), образовавшегося в результате поглощения, при расчете коэффициента $N$-фотонного поглощения света $\left(K^{(N)}\right)$ можно считать $\mathscr{N} \ll \mathbf{k}$ и положить $\mathscr{N}=0$. В пространственно однородном случае для поляризованного света матричные элементы оператора электрон-фотонного взаимодействия состоят из двух составляющих, одна из которых описывает два однофотонных взаимодействия, а вторая - взаимодействия электронов с двумя одновременно поглощающимися фотонами [3]. В этих условиях поглощения света линейной и циркулярной поляризации при расчетах составных матричных элементов $M_{n^{\prime} \mathbf{k}^{\prime}, n \mathbf{k}}^{(N)}$ $N$-фотонных переходов в методе эффективного гамильтониана $[17,18]$ достаточно рассматривать виртуальные состояния в пределах той же зоны $\Gamma_{8}^{+}$. Тогда, следуя [3], коэффициент $N$-фотонного поглощения можно записать в виде

$$
\begin{aligned}
K^{(N)}= & \frac{2 \pi}{\hbar} \frac{\hbar \omega}{I} \sum_{\mathbf{k}, m= \pm 1 / 2 ; m^{\prime}= \pm 3 / 2}\left(f_{1 \mathbf{k}}^{(N)}-f_{2 \mathbf{k}}^{(N)}\right)\left|M_{m ; m^{\prime}}^{(N)}(\mathbf{k})\right|^{2} \\
& \times \delta\left(E_{2 \mathbf{k}}-E_{1 \mathbf{k}}-N \hbar \omega\right),
\end{aligned}
$$

где $M_{m ; m^{\prime}}^{(N)}(\mathbf{k})$ - матричный элемент оптического перехода из состояния $|m \mathbf{k}\rangle$ в состояние $\left|m^{\prime} \mathbf{k}\right\rangle, \mathbf{k}-$ волновой 
вектор дырок, $I=\frac{n_{\omega} \omega^{2} A_{0}^{2}}{2 \pi c}-$ интенсивность света, $E_{l \mathbf{k}}-$ энергетический спектр дырок в подзоне $l: l=\mathrm{hh}$ или 1 $(l=\mathrm{lh}$ или 2$)$ для тяжелых (легких) дырок, $f_{l \mathbf{k}^{(N)}}-$ их неравновесная функция распределения при $N$-фотонном поглощении света, $n_{\omega}-$ коэффициент преломления света на частоте $\omega$. Остальные величины общеизвестные. Здесь электронам в подзоне $l=\mathrm{hh}$ (тяжелые дырки) соответствуют состояния с проекцией $m= \pm 3 / 2$ углового момента на направление $\mathbf{k}$, а электронам в подзоне $l=\mathrm{lh}$ (легкие дырки) - состояния с $m \pm 1 / 2$ [17,18].

Отметим, что далее однофотонный переход будем обозначать как $\left(m^{\prime}\right) \rightarrow(m)$, а двухфотонный как $\left(m^{\prime}\right) \Rightarrow(m)$, они отвечают матричным элементам $M_{m ; m^{\prime}}^{(1)}(k)$ и $M_{m ; m^{\prime}}^{(2)}(k)$ соответственно.

Тогда коэффициент многофотонного поглощения без учета когерентного насыщения определяется выражением $[3,5]$

$$
\begin{aligned}
K^{(N)}= & N \frac{2 \pi}{\hbar} \frac{\hbar \omega}{I}\left[\left|M_{+1 / 2 ;+3 / 2}^{(N)}(\mathbf{k})\right|^{2}+\left|M_{-1 / 2 ;+3 / 2}^{(N)}(\mathbf{k})\right|^{2}\right] \\
& \times \rho(N \hbar \omega) F(\beta, N, \omega),
\end{aligned}
$$

где $F(\beta, N, \omega)=\left[1-\exp (-N \hbar \omega) /\left(k_{\mathrm{B}} T\right)\right] \exp \left[\left(E_{\mathrm{F}}-E_{1}^{(N)}\right) /\right.$ $\left.k_{\mathrm{B}} T\right], E_{\mathrm{F}}$ - энергия Ферми, $E_{1}^{(N)}=N E_{1}^{*}, E_{1}^{*}=\frac{m_{\mathrm{hh}} m_{\mathrm{lh}}}{m_{\mathrm{hh}}-m_{\mathrm{lh}}} \hbar \omega$, $\rho(N \hbar \omega)=\mu_{-} k_{N} /\left(\pi^{2} \hbar^{2}\right)$ - приведенная плотность состояний фотовозбужденных дырок, $k_{N}-$ удовлетворяет уравнению $-2 B k_{N}^{2}=N \hbar \omega$, т.е. $k_{N}=\left(2 \mu_{-} N \omega / \hbar\right)^{1 / 2}$, $\mu_{-}=\frac{m_{\mathrm{hh}} m_{\mathrm{lh}}}{m_{\mathrm{hh}}-m_{\mathrm{lh}}}, m_{\mathrm{hh}}\left(m_{\mathrm{lh}}\right)-$ эффективные массы тяжелых (легких) дырок, $B=-\frac{\hbar^{2}}{2 \mu_{-}}-$зонный параметр полупроводника.

Из последнего соотношения видно, что для определения спектральной или температурной зависимости оптических параметров полупроводника, например $K^{(N)}$, нужно рассчитать матричные элементы рассматриваемых оптических переходов, которые мы будем анализировать далее для конкретных случаев.

Приведем сначала общую формулу для матричного элемента двухфотонных электронных переходов

$$
\begin{aligned}
M_{m \mathbf{k}, m^{\prime} \mathbf{k}}^{(2)}=M_{m, m^{\prime}}^{(2)}= & \sum_{m^{\prime \prime}= \pm 1 / 2, \pm 3 / 2} \frac{M_{m, m^{\prime \prime}}^{(1)} M_{m^{\prime \prime}, m^{\prime}}^{(1)}}{\left(E_{m^{\prime \prime} \mathbf{k}}-E_{m^{\prime} \mathbf{k}}-\hbar \omega\right)} \\
& -\left(\frac{e A_{0}}{c \hbar}\right)^{2}\left[H_{\Gamma_{8}}^{(2)}\left(\mathbf{e}^{\prime}\right)\right]_{m, m^{\prime}},
\end{aligned}
$$

где $H_{\Gamma_{8}}^{(2)}\left(\mathbf{e}^{\prime}\right)=H_{\Gamma_{8}}^{(2)}\left(\mathbf{k} \rightarrow \mathbf{e}^{\prime}\right), H_{\Gamma_{8}}^{(2)}(\mathbf{k})-$ эффективный гамильтониан дырок в представлении Латтинжера-Кона $[17,18]$; вектор е $\mathbf{e}^{\prime}$ имеет компоненты $e_{x^{\prime}}, e_{y^{\prime}}, e_{z^{\prime}}$; $e_{x^{\prime}}, e_{y^{\prime}}-$ проекции вектора поляризации света е на оси $x^{\prime}, y^{\prime}$, перпендикулярные волновому вектору $\mathbf{k}$. Заметим, что второе слагаемое (3) в методе эффективной массы описывает двухфотонные оптические переходы с виртуальными состояниями в других зонах [3], т.е. это слагаемое учитывает вклад в $M_{m \mathbf{k}, m^{\prime} \mathbf{k}}^{(2)}$ промежуточных состояний в других зонах; знак минус в этом слагаемом связан с тем, что в $\mathbf{k} \cdot \mathbf{p}$-методе энергетический знаменатель имеет вид типа $\left(E_{\Gamma_{8}}-E_{\tilde{n}}\right)$, a в остальном матричном элементе двухфотонного перехода аналогичный знаменатель записывается как $\left(E_{\Gamma_{8}}-E_{\tilde{n}}-\hbar \omega\right) \approx-\left(E_{\Gamma_{8}}-E_{\tilde{n}}\right)$ при $\left|E_{\Gamma_{8}}-E_{\tilde{n}}\right|\langle\hbar \omega$. Тогда матричный элемент этого оператора для переходов $(2, \mathbf{k}, m) \rightarrow\left(1, \mathbf{k}, m^{\prime}\right)$ в сферическом приближении равен

$$
\begin{aligned}
\widehat{\widehat{F}}_{1, m^{\prime}}^{+}(\mathbf{k}) H_{0} & \left(\frac{e}{c \hbar} \mathbf{A}\right) \widehat{F}_{2 m}(\mathbf{k}) \\
& =\left(\frac{e A_{0}}{c \hbar}\right)^{2}\left[-B \frac{m_{0}^{2}}{\hbar^{2}} \sum_{\alpha}\left(\widehat{J}_{\alpha} e_{\alpha}\right)^{2}\right]_{m^{\prime} m},
\end{aligned}
$$

где матрица-столбец $\widehat{F}_{l m}(\mathbf{k})$ - блоховская амплитуда волновой функции дырок в подзоне $(l, m, \mathbf{k})$ [17].

Поскольку нас интересуют оптические переходы типа $| \pm 3 / 2\rangle \mapsto| \pm 1 / 2\rangle$, далее приводим выражения для $N$-фотонного матричного элемента. При расчете $\left\|M_{m, m^{\prime}}^{(N)}\right\|$ учтены следующие фейнмановские диаграммы:

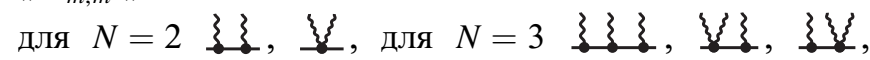

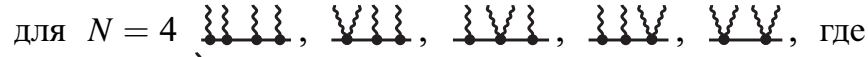
диаграмма $\}$ с соответствует однофотонному, а диаграмма $\{$ - одновременному поглощению двух фотонов. В частности, вклад в двухфотонный матричный элемент для оптического перехода типа $( \pm 3 / 2) \Rightarrow( \pm 1 / 2)$ равен $\sqrt{3} B m_{0}^{2} e_{z^{\prime}} e_{\mp}^{\prime} / \hbar$, а для переходов $( \pm 3 / 2) \Rightarrow(\mp 1 / 2)$ имеем $\frac{\sqrt{3}}{2} B m_{0}^{2}\left(e_{\mp}^{\prime}\right)^{2} / \hbar$. Это совпадает с результатами, получающимися при явном учете виртуальных состояний в зоне $\left(c, \Gamma_{6}\right)[5]$. Таким образом, нетрудно получить следующие выражения для абсолютного значения матричного элемента $M_{\mathbf{n}^{\prime} \mathbf{k}^{\prime}, \mathbf{n k}}^{(N)}$, полезные для расчета спектральной и температурной зависимости $K^{(N)}$

$$
\begin{aligned}
& \left\|M_{m, m^{\prime}}^{(2)}\right\|=\frac{\sqrt{3}}{2}\left(\frac{e A_{0}}{c \hbar}\right)^{2} B\left\|\begin{array}{cc}
6 e_{z}^{\prime} e_{-}^{\prime} & e_{-}^{\prime 2} \\
e_{+}^{\prime 2} & 6 e_{z}^{\prime} e_{+}^{\prime}
\end{array}\right\|, \\
& \left\|M_{m, m^{\prime}}^{(3)}\right\|=-\frac{\sqrt{3}}{2}\left(\frac{e A_{0}}{c \hbar}\right)^{3} \frac{B^{2} k_{3}}{\hbar \omega} \\
& \times\left\|\begin{array}{cc}
e_{+}^{\prime}\left(136 e_{z}^{\prime 2}-13 e_{\perp}^{\prime 2}\right) & 16 e_{z^{\prime}} e_{+}^{\prime 2} \\
-16 e_{z^{\prime}} e_{-}^{\prime 2} & e_{-}^{\prime}\left(136 e_{z}^{\prime 2}-13 e_{\perp}^{\prime 2}\right)
\end{array}\right\|, \\
& \left\|M_{m, m^{\prime}}^{(4)}\right\|=-\frac{\sqrt{3}}{6}\left(\frac{e A_{0}}{c \hbar}\right)^{4} \frac{B^{2}}{\hbar \omega} \frac{\left(B k^{2}\right)}{(\hbar \omega)^{2}} \\
& \times\left\|\begin{array}{cc}
4 e_{z^{\prime}} e_{-}^{\prime}\left(18 e_{z}^{\prime 2}-5 e_{\perp}^{\prime 2}\right) & 3 e_{+}^{\prime 2}\left(12 e_{z^{\prime}}^{2}-\left|e_{+}^{\prime}\right|^{2}\right) \\
-3 e_{-}^{\prime 2}\left(12 e_{z^{\prime}}^{2}-\left|e_{+}^{\prime}\right|^{2}\right) & 4 e_{z^{\prime}} e_{+}^{\prime}\left(18 e_{z}^{\prime 2}-5 e_{\perp}^{\prime 2}\right)
\end{array}\right\|,
\end{aligned}
$$

где $k_{3}=k_{N=3}, e_{ \pm}^{\prime}=e_{x^{\prime}} \pm e_{y^{\prime}}$.

Тогда с помощью формул (5)-(7), проводя в них угловое усреднение по телесным углам волнового вектора дырок (см. Приложение), получим соотношения для коэффициента ЛЦД для „парциальных“ оптических переходов: для трехфотонного поглощения $\eta^{(1+1+1)}=2.9$, 


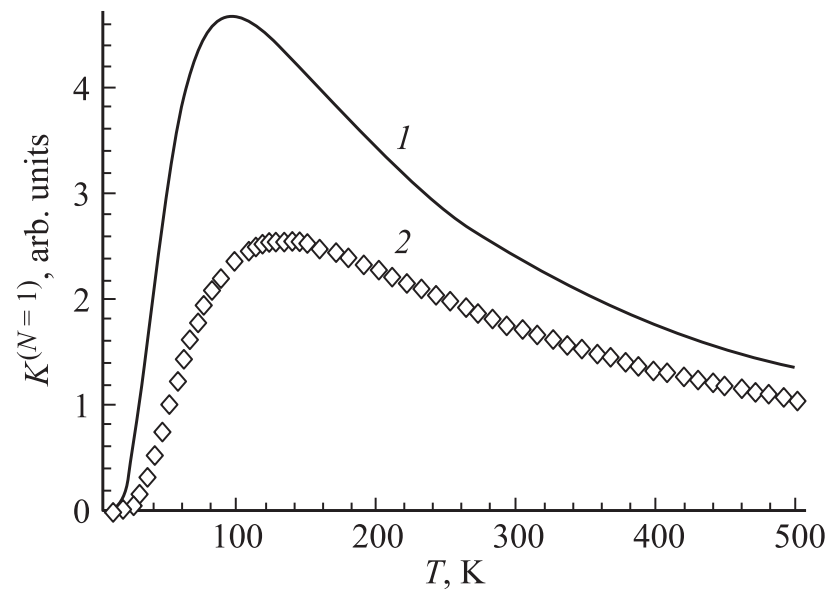

Температурная зависимость коэффициента однофотонного поглощения света без учета эффекта насыщения поглощения в $p$-GaAs для длин волн излучения $\lambda=9.5(1)$ и $10.6 \mu \mathrm{m}(2)$.

$\eta^{(1+2 ; 2+1)}=1.1$, а для четырехфотонного поглощения $\eta^{(1+1+1+1)}=1.1, \quad \eta^{(1+1+2 ; 1+2+1 ; 2+1+1)}=1.0, \quad \eta^{(2+2)}=2.6$, где верхние индексы описывают порядок поглощения по числу фотонов.

Для исследования температурной зависимости $K^{(N)}(\omega, T)$ запишем выражение для отношения коэффициентов $N^{\prime}$ и $N$ фотонного поглощения

$$
\frac{K^{\left(N^{\prime}\right)}}{K^{(N)}}=\sqrt{\frac{N^{\prime 3}}{N^{3}}} \Xi^{\left(N^{\prime}, N\right)} \exp \left[-\left(E_{1}^{\left(N^{\prime}\right)}-E_{1}^{(N)}\right) / k_{\mathrm{B}} T\right],
$$

где

$$
\Xi^{\left(N^{\prime}, N\right)}=\frac{\sum_{m^{\prime}= \pm 1 / 2, m= \pm 3 / 2}\left\langle\left|M_{m^{\prime} m}^{\left(N^{\prime}\right)}(\mathbf{k})\right|^{2}\right\rangle}{\sum_{m^{\prime}= \pm 1 / 2, m= \pm 3 / 2}\left\langle\left|M_{m^{\prime} m}^{(N)}(\mathbf{k})\right|^{2}\right\rangle} .
$$

В частности, в приближении $\hbar \omega\rangle\rangle k_{\mathrm{B}} T$ имеем

$$
\frac{K^{(4)}}{K^{(1)}}=4 \exp \left(-3 E_{1}^{(1)} / k_{\mathrm{B}} T\right) \Xi^{(4,1)},
$$

где

$$
\begin{aligned}
\Xi^{(4,1)}= & \frac{\sum_{m^{\prime}= \pm 1 / 2, m= \pm 3 / 2}\left\langle\left|M_{m^{\prime} m}^{(4)}(\mathbf{k})\right|^{2}\right\rangle}{\sum_{m^{\prime}= \pm 1 / 2, m= \pm 3 / 2}\left\langle\left|M_{m^{\prime} m}^{(1)}(\mathbf{k})\right|^{2}\right\rangle} \\
= & \frac{\varepsilon^{3}}{315}\left\{\begin{array}{l}
12298-\text { для линейной поляризации, } \\
11008-\text { для циркулярной поляризации, }
\end{array}\right.
\end{aligned}
$$

$\varepsilon=\frac{e A_{0}}{c \hbar} \sqrt{B}, K^{(1)}-$ коэффициент однофотонного поглощения света без учета эффекта насыщения поглощения $[2,3]$, в температурной зависимости которого имеется экстремальное значение (см. рисунок), определяемое соотношением

$$
T_{\mathrm{extr}}^{(N=1)}=\frac{2}{3} \frac{m_{\mathrm{lh}}}{m_{\mathrm{hh}}-m_{\mathrm{lh}}} \frac{\hbar \omega}{k_{\mathrm{B}}} .
$$

Например, для $p$-GaAs [4] при $\lambda=10.6 \mu$ m имеем $T_{\text {extr }}^{(N=1)}=194 \mathrm{~K}$.

Далее рассмотрим нелинейное поглощение света в полупроводнике, обусловленное вертикальными оптическими переходами между подзонами тяжелых и легких дырок валентной зоны и когерентным (резонансным) насыщением поглощения. 2 Это насыщение обусловлено возникновением фотоиндуцированной добавки в функции распределения дырок в поле сильной электромагнитной волны и приводит к дополнительному вкладу в коэффициент поглощения света.

Тогда в сферическом приближении в эффективном гамильтониане $[17,18]$ для дырок коэффициент многофотонного поглощения света $K^{(N)}(N=2,3,4)$ с учетом эффекта насыщения поглощения определяется выражением

$$
\begin{aligned}
K^{(N)}= & \frac{2 \pi}{\hbar} N \hbar \omega \frac{1}{I} \rho(N \hbar \omega) F(\beta, N, \omega) \\
& \times\left(\left\langle\frac{\left|M_{+3 / 2,+1 / 2}^{(N)}(\mathbf{k})\right|^{2}}{\sqrt{1+4 \frac{\alpha_{\omega}}{\hbar^{2} \omega^{2}}\left|M_{+3 / 2,+1 / 2}^{(N)}(\mathbf{k})\right|^{2}}}\right\rangle\right. \\
& \left.+\left\langle\frac{\left|M_{-3 / 2,-1 / 2}^{(N)}(\mathbf{k})\right|^{2}}{\sqrt{1+4 \frac{\alpha_{\omega}}{\hbar^{2} \omega^{2}}\left|M_{-3 / 2,-1 / 2}^{(N)}(\mathbf{k})\right|^{2}}}\right\rangle\right),
\end{aligned}
$$

где $\quad \alpha_{\omega}=6 \omega^{2} T_{1}^{(1)} T_{2}^{(1)} \frac{I}{I_{0}}, \quad I_{0}=\frac{c n_{\omega} \hbar^{3} \omega^{3}}{2 \pi|B|} . \quad$ Например, для $p$-GaAs и $I_{0}=13420 \mathrm{~kW} / \mathrm{cm}^{2}$ при $\hbar \omega=17 \mathrm{meV}$, $m_{2}=0.045 m_{0}$. Здесь пренебрегаем волновым вектором фотона, т. е. считаем, что $k^{\prime}=k_{N} \cong k(n=1,2,3,4)$.

Из последнего соотношения видно, что резонансное насыщение приводит к уменьшению коэффициента поглощения с ростом интенсивности излучения $I$ по закону $\sqrt{I}$.

Отметим здесь, что в [3-7] показано, что при малой интенсивности излучения, когда фотоиндуцированным изменением функции распределения дырок можно пренебречь, суммарные вероятности для оптических переходов $(+3 / 2, \mathbf{k}) \rightarrow(+1 / 2, \mathbf{k}) \quad$ и $(-3 / 2, \mathbf{k}) \rightarrow(-1 / 2, \mathbf{k})$ не зависят от степени поляризации света. C учетом эффекта насыщения, как видно из (11), эти вероятности нужно умножить соответственно на $\sqrt{1+4 \frac{\alpha_{\omega}}{\hbar^{2} \omega^{2}}\left|M_{ \pm 3 / 2, \pm 1 / 2}^{(N, \operatorname{lin})}(\mathbf{k})\right|^{2}} \quad$ и $\sqrt{1+4 \frac{\alpha_{\omega}}{\hbar^{2} \omega^{2}}\left|M_{ \pm 3 / 2, \pm 1 / 2}^{(N, \text { circ })}(\mathbf{k})\right|^{2}}$, за счет этого суммарная вероятность соответствующих переходов будет зависеть от состояния поляризации света.

Также отметим, что при многофотонных переходах промежуточные состояния дырок являются виртуальными. Поэтому нельзя пренебречь интерференцией вкладов в многоквантовый процесс $(m, \mathbf{k}) \rightarrow\left(m^{\prime}, \mathbf{k}\right)$

\footnotetext{
${ }^{2}$ В [3-6] механизмом нелинейности поглощения считается резонансное насыщение однофотонного поглощения.
} 
$\rightarrow\left(m^{\prime \prime}, \mathbf{k}\right) \rightarrow\left(m^{\prime \prime \prime}, \mathbf{k}\right) \rightarrow \ldots(l, \mathbf{k})$ от различных виртуальных состояний $\left(m^{\prime}, \mathbf{k}\right),\left(m^{\prime \prime}, \mathbf{k}\right), \quad\left(m^{\prime \prime \prime}, \mathbf{k}\right), \ldots$ Это означает, что недостаточно произвести суммирование по $\quad m^{\prime}, m^{\prime \prime}, m^{\prime \prime \prime}, \ldots \quad$ величины $\quad\left|M_{m, m^{\prime}, m^{\prime \prime}, m^{\prime \prime \prime} \ldots l}(\mathbf{k})\right|^{2}$. Сначала следует просуммировать по $m, m^{\prime}, m^{\prime \prime}, m^{\prime \prime \prime}, \ldots$ матричным элементам многофотонных переходов $\left|M_{m, m^{\prime}, m^{\prime \prime}, m^{\prime \prime \prime} \ldots l}(\mathbf{k})\right|^{2}$, а затем возвести в квадрат модуль полученной суммы.

Таким образом, вклад в коэффициент многофотонного поглощения эффекта насыщения определяется как

$$
\begin{aligned}
& \delta K^{(N)} \propto \sum_{m^{\prime}= \pm 1 / 2, m= \pm 3 / 2} \delta\left|M_{m^{\prime} m}^{(N)}(\mathbf{k})\right|^{2} \\
& =\sum_{m^{\prime}= \pm 1 / 2, m= \pm 3} \frac{\left|M_{m^{\prime} m}^{(N)}(\mathbf{k})\right|^{2}}{\sqrt{1+4 \frac{\alpha_{\omega}}{\hbar^{2} \omega^{2}}\left|M_{m^{\prime} m}^{(N)}(\mathbf{k})\right|^{2}}}-\left|M_{m^{\prime} m}^{(N)}(\mathbf{k})\right|^{2}
\end{aligned}
$$

Далее рассмотрим область умеренной интенсивности, где можно использовать теорию возмущений. Тогда, разлагая радикал в (12) в ряд по $4 \frac{\alpha_{\omega}}{\hbar^{2} \omega^{2}}\left|M_{m^{\prime} m}^{(N)}(\mathbf{k})\right|^{2}$, получаем следующие результаты.

При $N=1$ имеем следующее полезное для дальнейшего анализа результатов выражение:

$$
\begin{aligned}
\delta\left|M_{m^{\prime} m}^{(N=1)}(\mathbf{k})\right|^{2}= & -\frac{1}{2} \cdot 4 \frac{\alpha_{\omega}}{\hbar^{2} \omega^{2}}\left|M_{m^{\prime} m}^{(1)}(\mathbf{k})\right|^{4} \\
& +\frac{1 \cdot 3}{2 \cdot 4}\left(4 \frac{\alpha_{\omega}}{\hbar^{2} \omega^{2}}\right)^{2}\left|M_{m^{\prime} m}^{(1)}(\mathbf{k})\right|^{6} \\
& -\frac{1 \cdot 3 \cdot 5}{2 \cdot 4 \cdot 6}\left(4 \frac{\alpha_{\omega}}{\hbar^{2} \omega^{2}}\right)^{3}\left|M_{m^{\prime} m}^{(1)}(\mathbf{k})\right|^{8}+\ldots
\end{aligned}
$$

где первое слагаемое дает линейный, второе - квадратичный, третье - кубический по интенсивности вклад в коэффициент поглощения (ЛЦД). Тогда

$$
\begin{gathered}
\sum_{m^{\prime}= \pm 1 / 2, m= \pm 3 / 2}\left\langle 2 \frac{\alpha_{\omega}}{\hbar^{2} \omega^{2}}\left(\left|M_{m^{\prime} m}^{(1)}(\mathbf{k})\right|^{2}\right)^{2}\right\rangle \\
=18 \frac{\alpha_{\omega}}{\hbar^{2} \omega^{2}}\left(\frac{e A_{0}}{c \hbar}\right)^{4} B^{4} k^{4}\left\langle\left|e_{\mp}^{\prime}\right|^{4}\right\rangle \\
\sum_{m^{\prime}= \pm 1 / 2, m= \pm 3 / 2}\left\langle\frac{3}{8}\left(4 \frac{\alpha_{\omega}}{\hbar^{2} \omega^{2}}\right)^{2}\left(\left|M_{m^{\prime} m}^{(1)}(\mathbf{k})\right|^{2}\right)^{3}\right\rangle \\
=162\left(\frac{\alpha_{\omega}}{\hbar^{2} \omega^{2}}\right)^{2}\left(\frac{e A_{0}}{c \hbar}\right)^{6} B^{6} k^{6}\left\langle\left|e_{\mp}^{\prime}\right|^{6}\right\rangle \\
\left\langle\frac{5}{16}\left(4 \frac{\alpha_{\omega}}{\hbar^{2} \omega^{2}}\right)^{2}\left(\left|M_{m^{\prime} m}^{(1)}(\mathbf{k})\right|^{2}\right)^{4}\right\rangle \\
=1620\left(\frac{\alpha_{\omega}}{\hbar^{2} \omega^{2}}\right)^{2}\left(\frac{e A_{0}}{c \hbar}\right)^{8} B^{8} k^{8}\left\langle\left|e_{\mp}^{\prime}\right|^{8}\right\rangle
\end{gathered}
$$

Проводя угловое усреднение и используя обозначение $\beta_{\omega}=\alpha_{\omega}\left(\frac{e A_{0}}{c \hbar}\right)^{2} \frac{B}{\hbar \omega}$, перепишем последние выражения как

$$
-\left\langle\frac{\alpha_{\omega}}{\hbar^{2} \omega^{2}}\left(\left|M_{m^{\prime} m}^{(1)}(\mathbf{k})\right|^{2}\right)^{2}\right\rangle=-\frac{1}{315} \alpha_{\omega}\left(\frac{e A_{0}}{c \hbar}\right)^{4}
$$$$
\times B^{2}\left\{\begin{array}{l}
1512 \text { - для линейной поляризации, } \\
1323 \text { - для циркулярной поляризации, }
\end{array}\right.
$$

$$
\begin{aligned}
& \left\langle\frac{3}{8}\left(4 \frac{\alpha_{\omega}}{\hbar^{2} \omega^{2}}\right)^{2}\left(\left|M_{m^{\prime} m}^{(1)}(\mathbf{k})\right|^{2}\right)^{3}\right\rangle=\frac{1}{315} \alpha_{\omega}\left(\frac{e A_{0}}{c \hbar}\right)^{4} \\
& \times B^{2} \beta_{\omega}\left\{\begin{array}{l}
23328-\text { для линейной поляризации, } \\
17496-\text { для циркулярной поляризации, }
\end{array}\right. \\
& \left\langle-\frac{5}{16}\left(4 \frac{\alpha_{\omega}}{\hbar^{2} \omega^{2}}\right)^{3}\left(\left|M_{m^{\prime} m}^{(1)}(\mathbf{k})\right|^{2}\right)^{4}\right\rangle=-\frac{1}{315} \alpha_{\omega}\left(\frac{e A_{0}}{c \hbar}\right)^{4} \\
& \times B^{2} \beta_{\omega}\left\{\begin{array}{l}
207360-\text { для линейной поляризации, } \\
134460-\text { для циркулярной поляризации, }
\end{array}\right.
\end{aligned}
$$

Из последних соотношений видно, что каждое слагаемое (13) вносит вклад в суммарный коэффициент поглощения (ЛЦД).

Вклад двухфотонного эффекта насыщения в поглощение определим как

$$
\begin{aligned}
& \sum_{m^{\prime}= \pm 1 / 2, m= \pm 3 / 2}\left\langle\delta\left|M_{m^{\prime} m}^{(N=2)}(\mathbf{k})\right|^{2}\right\rangle=-\frac{9}{4} \frac{\alpha_{\omega}}{\hbar^{2} \omega^{2}}\left(\frac{e A_{0}}{c \hbar}\right)^{8} \\
& \times B^{4}\left\langle\left(\left|e_{ \pm}^{\prime 2}\right|^{4}+1296\left|e_{+}^{\prime} e_{z^{\prime}}\right|^{4}\right)\right\rangle=\frac{1}{315} \frac{\alpha_{\omega}}{\hbar^{2} \omega^{2}}\left(\frac{e A_{0}}{c \hbar}\right)^{8} \\
& \times B^{4}\left\{\begin{array}{l}
23616-\text { для линейной поляризации, } \\
36011-\text { для циркулярной поляризации. }
\end{array}\right.
\end{aligned}
$$

Тогда коэффициент ЛЦД для данного случая $\delta \eta^{(N=2)}=0.7$.

В заключение отметим следующее:

1) вероятности многофотонных переходов зависят от степени поляризации света, т.е. имеет место линейноциркулярный дихроизм нелинейного по интенсивности поглощения света в полупроводнике со сложной валентной зоной;

2) температурная зависимость коэффициента $N$-фотонного межподзонного поглощения поляризованного излучения в области частот, где $\hbar \omega \gg k_{\mathrm{B}} T$, определяется температурной зависимостью коэффициента однофотонного поглощения, которую следует умножить на $\exp \left[-(N-1) \frac{m_{\mathrm{lh}}}{m_{\mathrm{hh}}-m_{\mathrm{lh}}} \frac{\hbar \omega}{k_{\mathrm{B}} T}\right]$;

3) физическая природа выстраивания импульса фотовозбужденных дырок и ориентация их моментов зависят 
Выражения для средних величин $e_{\alpha}^{\prime m}$ и $e_{\beta}^{\prime n}$ при разных значениях $m$ и $n$ и различных поляризациях света $(\alpha, \beta=x, y, z)$

\begin{tabular}{|c|c|c|}
\hline $\begin{array}{c}\text { Усредняющие } \\
\text { величины }\end{array}$ & $\begin{array}{c}\text { Линейная } \\
\text { поляризация }\end{array}$ & $\begin{array}{c}\text { Циркулярная } \\
\text { поляризация }\end{array}$ \\
\hline$\overline{\mid \overline{\left|e_{+}^{\prime}\right|^{2}}}$ & $2 / 3$ & $2 / 3$ \\
\hline$\overline{\left|e_{+}^{\prime}\right|^{4}}$ & $8 / 15$ & $7 / 15$ \\
\hline$\overline{\left|e_{+}^{\prime}\right|^{6}}$ & $16 / 35$ & $12 / 35$ \\
\hline$\overline{\left|e_{+}^{\prime}\right|^{8}}$ & $128 / 315$ & $83 / 315$ \\
\hline$\overline{e_{z}^{\prime 2}}$ & $1 / 3$ & $1 / 3$ \\
\hline$\overline{e_{z}^{\prime 4}}$ & $1 / 5$ & $2 / 15$ \\
\hline$\overline{e_{z}^{\prime 4}}$ & $1 / 7$ & $2 / 35$ \\
\hline$\overline{e_{z}^{\prime 8}}$ & $1 / 9$ & $8 / 315$ \\
\hline$\overline{\left|e_{+}^{\prime}\right|^{2} e_{z}^{\prime 2}}$ & $2 / 15$ & $1 / 5$ \\
\hline$\overline{\left|e_{+}^{\prime}\right|^{2} e_{z}^{\prime 4}}$ & $2 / 35$ & $8 / 105$ \\
\hline$\overline{\left|e_{+}^{\prime}\right|^{4} e_{z}^{\prime 2}}$ & $8 / 105$ & $13 / 105$ \\
\hline$\overline{\left|e_{+}^{\prime}\right|^{4} e_{z}^{\prime 4}}$ & $8 / 315$ & $2 / 45$ \\
\hline$\overline{\left|e_{+}^{\prime}\right|^{2} e_{z}^{\prime 6}}$ & $2 / 63$ & $2 / 63$ \\
\hline$\overline{\left|e_{+}^{\prime}\right|^{6} e_{z}^{\prime 2}}$ & $16 / 315$ & $5 / 63$ \\
\hline
\end{tabular}

от выбора модели задачи, например от типов оптических переходов.

Исследование многофотонного линейно-циркулярного дихроизма в размерно-квантованных структурах $[19,20]$, где оптические переходы протекают в два этапа, можно произвести аналогичным образом. На первом этапе происходят переходы в двумерном импульсном пространстве, как в объемном полупроводнике, а на втором этапе происходят переходы между размерно-квантованными состояниями. Естественно, каждый из этих этапов имеет свое правило отбора оптических переходов.

Физическая природа неодновременного поглощения двух или более фотонов в таких структурах зависит от выбора модели задачи, например от структуры зоны полупроводника или от направления выращивания структуры.

Эти случаи требуют более подробного рассмотрения, которому будет посвящена отдельная работа.

\section{Приложение}

Здесь остановимся на угловых усреднениях по всем направлениям волнового вектора дырки k. При этом используем следующую геометрию: выберем проекции вектора поляризации света $\mathbf{e}^{\prime}=\left\{e_{x}^{\prime}, e_{y}^{\prime}, e_{z}^{\prime}\right\}$ на оси $x^{\prime}, y^{\prime}, z^{\prime}$, связанные с направлением вектора $\mathbf{k}\left(\mathbf{k} \uparrow \uparrow z^{\prime}\right)$, ось $x^{\prime}$ лежит в плоскости $(\mathscr{N}, \mathbf{k}) ; \theta-$ угол между векторами $\mathscr{N}$ и $\mathbf{k}$.

Тогда угловое усреднение дает полезные для дальнейших расчетов выражения (см. таблицу).

Далее также приводим часто используемые формулы

$$
\frac{1}{4 \pi} \int_{0}^{\pi} \sin \theta d \theta \int_{0}^{2 \pi} d \varphi(\mathbf{a} \cdot \mathbf{o})(\mathbf{b} \cdot \mathbf{o})=\frac{1}{3}(\mathbf{a} \cdot \mathbf{b}),
$$

$$
\begin{aligned}
& \frac{1}{4 \pi} \int_{0}^{\pi} \sin \theta d \theta \int_{0}^{2 \pi} d \varphi(\mathbf{a} \cdot \mathbf{o})(\mathbf{b} \cdot \mathbf{o})(\mathbf{c} \cdot \mathbf{o})(\mathbf{d} \cdot \mathbf{o}) \\
& \quad=\frac{1}{15}[(\mathbf{a} \cdot \mathbf{b})(\mathbf{c} \cdot \mathbf{d})+(\mathbf{a} \cdot \mathbf{c})(\mathbf{d} \cdot \mathbf{b})+(\mathbf{a} \cdot \mathbf{d})(\mathbf{c} \cdot \mathbf{b})],
\end{aligned}
$$

где о $(\theta, \varphi)$ - единичный вектор в направлении, определяемом углами $\theta$ и $\varphi ; \mathbf{a}, \mathbf{b}, \mathbf{c}, \mathbf{d}-$ произвольные постоянные векторы.

\section{Список литературы}

[1] Е.Л. Ивченко. ФТТ 14, 3489 (1972).

[2] Р.Я. Расулов. Докт. дис. ФТИ им. А.Ф. Иоффе РАН, СПб. (1993). Гл. 3.

[3] С.Д. Ганичев, Е.Л. Ивченко, Р.Я. Расулов, И.Д. Ярошецкий, Б.Я. Авербух. ФТТ 35, 198 (1993); Р.Я. Расулов. ФТТ 35, 1107 (1993).

[4] Д.А. Паршин, А.Р. Шабаев. ЖЭТФ 92, 1471 (1987).

[5] С.Д. Ганичев, С.А. Емельянов, Е.Л. Ивченко, Е.Ю. Перлин, Я.В. Терентьев, А.В. Федоров, И.Д. Ярошецкий. ЖЭТФ 91, 729 (1986).

[6] Р.Я. Расулов, Г.Х. Хошимов, Х. Холитдинов. ФТП 30, 274, (1996).

[7] Р.Я. Расулов. ФТП 22, 2077 (1988).

[8] Р.Я. Расулов. ФТТ 35, 1674 (1993).

[9] S. Krishnamurthi, Z.G. Yu, L.P. Gonzalez, S. Guha. J. Appl. Phys. 109, 033102 (2011).

[10] R.A. Negres, J.M. Hales, A. Kobyakov, D.J. Hagan, E.W. Van Stryland. IEEE J. Quantum Electron. 38, 1205 (2002).

[11] P.D. Olszak. Ph D Dissertation. College of Optics and Photonics, University of Central Florida, Orlando, Florida (2010). 143 p.

[12] J. López Gondar, R. Cipolatti, G.E. Marques. Brazil. J. Phys. 36, 960 (2006).

[13] N.R. Kulish, M.P. Lisitsa, N.I. Malysh. Semicond. Phys. Quantum Electron. Optoelectron. 8, 72 (2005).

[14] J. He, Y.Qu, H.Li, J. Mi, W. Ji. Opt. Express 13, 9235 (2005).

[15] W.C. Hurlbut, Y.S. Lee, K.L. Vodopyanov, P.S. Kuo, M.M. Fejer. Opt. Lett. 32, 668 (2007).

[16] S. Pearl, N. Rotenberg, H.M. van Driel. Appl. Phys. Lett. 93, 131102 (2008).

[17] Г.Л. Бир, Г.Е. Пикус. Симметрия и деформационные эффекты в полупроводниках. Наука, М. (1973). 584 с.

[18] Е.Л. Ивченко, Р.Я. Расулов. Симметрия и реальная зонная структура полупроводников. Фан, Ташкент (1989). 126 с.

[19] E.L. Ivchenko. Optical spectroscopy of semiconductor nanostructures. Alpha Science International, Ltd., Harrow (2005). Ch. XII. 427 p.

[20] G.E. Pikus, E.L. Ivchenko. Superlattices and other heterostructures: symmetry and optical phenomena. Springer-Verlag, Berlin-Heidelberg (1995). $657 \mathrm{p}$. 